\title{
Atención odontológica a pacientes con discapacidad mental y psicomotriz en la Facultad de Odontología de Mexicali*
}

Dental Care to Patients with Mental and Psychomotor Disabilities at Mexicali's Dental

School

Atendimento odontológico a pacientes com deficiência mental e psicomotora na Faculdade de

Odontologia de Mexicali

\author{
Norma Patricia Figueroa FernándeZ ${ }^{a}$ \\ Universidad Autónoma de Baja California. Mexicali, México. nfigueroa@uabc.edu.mx. \\ https://orcid.org/0000-0003-2318-4538
}

\begin{abstract}
MAIKel Hermida Rojas
Universidad Autónoma de Baja California. Mexicali, México. maikel.hermida@uabc.edu.mx. https://orcid.org/0000-0002-5730-7068
\end{abstract}

\begin{abstract}
ANITZA Domínguez SÁNCHEZ
Universidad Autónoma de Baja California. Mexicali, México. anitzadominguez@uabc.edu.mx.

https://orcid.org/0000-0002-4020-1697
\end{abstract}

IRMA IRENE ZORRILLA MARTÍNEZ 
Universidad Autónoma de Baja California. Mexicali, México. irene.zorrilla@uabc.edu.mx. https://orcid.org/0000-0003-3461-7212

\section{YANETH YAdiRa VALENZUELA ONTIVEROS}

Universidad Autónoma de Baja California. Mexicali, México. valenzuela.yanteh@uabc.edu.mx. https://orcid.org/0000-0002-2147-4577

\section{FEDERICO RIVERA LUNA}

Universidad Autónoma de Baja California. Mexicali, México. federicorivera@uabc.edu.mx. https://orcid.org/0000-0003-0913-7698

*Investigación original

${ }^{a}$ Correspondencia: nfigueroa@uabc.edu.mx

doi: https://doi.org/10.11144/Javeriana.uo38-81.aopd

Cómo citar: Figueroa Fernández NP, Hermida Rojas M, Domínguez Sánchez A, Zorrilla Martínez II, Valenzuela OntiverosYY, Rivera Luna F. Atención odontológica a pacientes con discapacidad mental y psicomotriz en la Facultad de Odontología de Mexicali. Univ Odontol. 2019 jun-dic; 38(81). https://doi.org/10.11144/Javeriana.uo38-81.aopd

\section{RESUMEN}


Antecedentes: Las enfermedades bucales en pacientes con discapacidad implican procesos complejos tanto en atención como en comunicación, por lo que el tratamiento odontológico bajo anestesia general es excelente opción. Objetivo: Describir las experiencias en atención odontológica bajo anestesia general a pacientes con discapacidad mental y psicomotriz registrados en las clínicas de la Facultad de Odontología Mexicali (FOM). Método: Estudio observacional, analítico, transversal, sobre aspectos de atención odontológica, con datos disponibles en expedientes clínicos de todos los pacientes intervenidos bajo anestesia general, en el quirófano de la FOM, durante 2016 y 2017, los datos fueron vaciados para su análisis y descripción en el paquete estadístico STATA12. Resultados: La muestra quedó conformada por 38 casos, el sexo masculino predominó sobre el femenino (68 \% vs $32 \%$ ). La media de edad fue $15.71 \pm 8.75$ años, la caries dental fue la necesidad de atención de mayor frecuencia, seguida de traumatismos dentoalveolares, y enfermedad periodontal. En el análisis bivariado, se encontró asociación significativa entre las complicaciones y el tiempo de duración del procedimiento con $X^{2}=7.05$ y un valor de $p=0.029$. Conclusión: Predominó la discapacidad psicomotriz, con mayor incidencia en menores de 10 años y en el sexo masculino. Todos los pacientes presentaron caries dental, que se acompañaba de fractura dentoalveolar en más de un tercio de los casos, por lo que hubo alta necesidad de tratamientos quirúrgicos. La frecuencia de complicaciones derivadas de la anestesia general fueron pocas y estuvieron asociadas al tiempo de exposición a la anestesia general.

\section{Palabras clave}


atención dental para personas con discapacidad; discapacidad; discapacidad intelectual; personas con discapacidad; odontología; salud oral; personas con discapacidad mental; salud de personas con discapacidad; servicios de salud para personas con discapacidad

\section{ABSTRACT}

Background: Oral diseases in patients with disabilities involve complex processes in both attention and communication, so dental treatment under general anesthesia is an excellent option. Purpose: To describe the experiences in dental care under general anesthesia to patients with mental and psychomotor disabilities registered in the clinics of the Faculty of Dentistry Mexicali (FOM). Methods: Observational, analytical, cross-sectional study on aspects of dental care, with data available in clinical records of all patients operated under general anesthesia, in the FOM operating room, during 2016 and 2017, the data were emptied for analysis and description in the statistical package STATA12. Results: The sample was conformed by 38 cases, the masculine sex prevailed over the feminine one ( $68 \%$ vs $32 \%)$. The mean age was $15.71 \pm 8.75$ years, dental caries was the need for more frequent care, followed by dentoalveolar trauma, and periodontal disease. In the bivariate analysis, a significant association was found between complications and the duration of the procedure with $\mathrm{X} 2=7.05$ and a value of $\mathrm{p}=0.029$. Conclusion: Psychomotor disability predominated, with a greater incidence in children under 10 years of age and in the male sex. All patients had dental caries, which was accompanied by dentoalveolar fracture in more than a third of the cases, so there was a high need for surgical treatments. The frequency of complications derived from general anesthesia were few and were associated with the time of exposure to general anesthesia. 


\section{Keywords}

dental care for people with disabilities; dentistry; health of people with disabilities; health services for people with disabilities; intellectual disability; oral health; people with disabilities; people with mental disabilities

\section{RESUMO}

Antecedentes: As doenças bucais em pacientes com deficiência envolvem processos complexos tanto na atenção quanto na comunicação, portanto, o tratamento odontológico sob anestesia geral é uma excelente opção. Objetivo: Descrever as experiências em atendimento odontológico sob anestesia geral a pacientes com deficiência mental e psicomotora cadastrados nas clínicas da Faculdade de Odontologia Mexicali (FOM). Método: Estudo observacional, analítico e transversal sobre aspectos do atendimento odontológico, com dados disponíveis nos prontuários de todos os pacientes operados sob anestesia geral, na sala de operações da FOM, durante 2016 e 2017, os dados foram esvaziados para análise e descrição no pacote estatístico STATA12. Resultados: A amostra foi conformada por 38 casos, o sexo masculino prevaleceu sobre o feminino (68\% vs $32 \%$ ). A idade média foi de 15,71 $\pm 8,75$ anos, a cárie dentária foi a necessidade de cuidados mais frequentes, seguidos de trauma dentoalveolar e doença periodontal. Na análise bivariada, foi encontrada associação significativa entre as complicações e a duração do procedimento com X2 = 7,05 e um valor de $\mathrm{p}=0,029$. Conclusão: A incapacidade psicomotora predominou, com maior incidência em crianças menores de 10 anos e no sexo masculino. Todos os pacientes apresentaram cárie dentária, 
que foi acompanhada por fratura dentoalveolar em mais de um terço dos casos, havendo grande necessidade de tratamentos cirúrgicos. A frequência de complicações derivadas da anestesia geral foi pequena e esteve associada ao tempo de exposição à anestesia geral.

\section{Palavras Chave}

assistência odontológica a pessoas com deficiencia; deficiência intelectual; Incapacidade; odontología; saúde bucal; pessoas com deficiencia; pessoas com deficiência mental; saúde das pessoas com deficiencia; serviços de saúde para pessoas com deficiência

\section{INTRODUCCIÓN}

La Organización Mundial de la Salud (OMS) considera a la discapacidad como un término genérico que abarca diversas deficiencias, limitaciones de la actividad y restricciones de participación social de quien la padece (1). Este mismo organismo realizó la Clasificación Internacional del Funcionamiento de la Discapacidad y de la Salud, donde se agrupan las alteraciones funcionales y/o estructurales del cuerpo, con repercusiones totales o parciales; además, de que definió los términos: "limitación de actividad" como la dificultad para ejecutar una tarea o acción, y "restricción de participación" que es el problema experimentado por el individuo involucrado en situaciones de la vida (2).

En México, la Secretaría de Salud estableció la Norma Oficial Mexicana NOM-015-SSA3-2012, para regular la atención integral a personas con discapacidad. En este documento legal se conceptualizan a las discapacidades como "deficiencia auditiva, intelectual, neuromotora o visual, 
ya sea de naturaleza permanente o temporal, que limita la capacidad de realizar una o más actividades de la vida diaria" (3). Por consiguiente, una persona con discapacidad es aquella que tenga deficiencias auditivas, intelectuales, neuromotoras o visuales que, al interactuar con diversas barreras, puedan impedir su participación plena y efectiva en la sociedad, en igualdad de condiciones con las demás personas.

En el contexto mexicano, el Instituto Nacional de Geografía e Informática (INEGI) concentró las discapacidades en cuatro grupos:

- Grupo 1.- Discapacidades sensoriales y de comunicación, que incluyen las oculares, las auditivas y del habla.

- Grupo 2.- Motrices: alteraciones de marcha, manipulación de objetos y coordinación de movimientos.

- Grupo 3.- Mentales: deficiencias intelectuales y conductuales que repercuten en el aprendizaje y en las formas de socialización.

- Grupo 4.- Múltiples y otras: abarca las combinaciones de los grupos descritos; así como las no consideradas en estos, como los síndromes que implican discapacidades múltiples, las causadas por deficiencias cardiacas, pulmonares, renales y enfermedades crónicas y/o degenerativas avanzadas (4).

De acuerdo con esta clasificación, las discapacidades pueden manifestarse de diversas formas y se dividen en categorías y niveles de compromiso orgánico o sistémico. Esta complejidad se relaciona con su etiología, que es multifactorial, donde se combinan factores intrínsecos y extrínsecos, y como resultado, los daños pueden ser estructurales y funcionales con diversas manifestaciones clínicas. 
La OMS ha señalado que las tasas de incidencia y prevalencia de las discapacidades tienden al aumento, como consecuencia del incremento de los nacimientos en países subdesarrollados, el embarazo tardío en los países desarrollados, la exposición a factores de riesgo que atentan contra la calidad de vida, el envejecimiento poblacional y la alta incidencia de enfermedades crónicas degenerativas. El Informe Mundial sobre Discapacidad 2010 de la OMS (IMD-2010), estimó que cerca del $15 \%$ de la población mundial vive con algún tipo de discapacidad y esto representa más de mil millones de personas (5).

En México, según los datos de la Encuesta Nacional de la Dinámica Demográfica (ENADID 2014), 7.1 millones de habitantes sufren algún tipo de discapacidad y esto representa el 6\% de la población. Los estados más afectados son Nayarit (8.2 por cada 1000 habitantes), Durando (75 por cada 1000 habitantes), Colima, Jalisco y Zacatecas (74 por cada 1000 habitantes). Mientras que, en el estado de Baja California, la tasa es de 58 discapacitados por cada 1000 habitantes y esto significa que un $5.8 \%$ de la población del estado padece alguna discapacidad (6).

El estado de salud es muy vulnerable en las personas con discapacidad y, cuando aparecen otras enfermedades, estos pacientes se enfrentan a diversas barreras para acceder a los servicios de salud. Las discapacidades se acompañan de limitación motora, sensorial, cognitiva, conductual y/o emocional, congénita o adquirida; y, como consecuencia, requiere de una intervención, manejo médico o asistencial especializado para recuperar y conservar su salud (7). Lamentablemente, la Encuesta Mundial de Salud (2009) señaló que las personas con discapacidad se enfrentan a 
problemas actitudinales, barreras físicas y condiciones sistémicas que interfieren en el acceso a su atención (8).

La salud oral es inseparable de la salud general, por lo que las enfermedades bucales tienen un impacto directo en la salud general y en la calidad de vida, especialmente en aquellos que tienen compromiso del sistema inmunológico (9). Las enfermedades bucales de los pacientes con discapacidad son las mismas que las de la población general, suelen presentarse de forma más severa, por la deficiente higiene oral derivada de la propia discapacidad y/o de los efectos en los tejidos orales de los fármacos prescritos debido a su condición. Al mismo tiempo, para su atención en el área de la Odontología, se requieren maniobras, conceptos, equipamiento y personal especializado para promover y prevenir las enfermedades bucodentales, así como garantizar su curación y total recuperación (10).

La atención odontológica a los pacientes con discapacidad implica procesos muy complejos de comunicación con el paciente y su familia, familiarización con el medio odontológico, diagnóstico y tratamiento multi e interdisciplinario. Dada la escasa cooperación y a la severidad de las enfermedades bucodentales del paciente con discapacidad motora y psicomotriz, por lo general se requiere de procedimientos múltiples y de larga duración; en estos casos, el uso de anestesia general es la mejor alternativa para la atención odontológica (11).

Ante la concurrencia de pacientes con discapacidad mental y psicomotriz a los servicios odontológicos universitarios del municipio Mexicali, en Baja California, se les ofrece atención clínica y quirúrgica según las recomendaciones de atención médica y odontológica a pacientes con 
necesidades especiales. Estos servicios asistenciales se iniciaron desde el año 2003 y se formalizaron en el año 2012, ante la apertura del "Programa de Atención Odontológica a Pacientes con Discapacidad" (12). En este programa, se ofrece atención odontológica integral especializada a pacientes con discapacidad, a través de docentes, pasantes y alumnos en etapa terminal de la Facultad de Odontología Mexicali (FOM), en la Universidad Autónoma de Baja California (UABC), para mejorar su calidad de vida a través de la conservación de la integridad del complejo bucal, mediante los lineamientos, las pautas terapéuticas y guías de atención médica y odontológica para personas con necesidades especiales, según la literatura científica contemporánea (13-16).

De acuerdo con los informes quirúrgicos y los reportes clínicos del "Programa de Atención Odontológica a Pacientes con Discapacidad", registrados en los expedientes de la FOM, se cuenta con evidencia del alcance y el impacto del tratamiento a pacientes con discapacidad mental y psicomotriz. Ante toda esa información clínico - terapéutica, surgió la siguiente pregunta de investigación: ¿Qué resultados medibles se han derivado de la atención odontológica a pacientes con discapacidad mental y psicomotriz, atendidos bajo anestesia general, en la Facultad de Odontología Mexicali? Para dar respuesta a esta interrogante, se realizó la presente investigación con el objetivo de describir los principales cuadros clínicos odontológicos y las pautas terapéuticas, bajo anestesia general, en la atención a pacientes con discapacidad mental y psicomotriz registrados en las clínicas de la Facultad de Odontología Mexicali.

\section{MATERIALES Y MÉTODOS}


Se realizó un estudio observacional, analítico y transversal, sobre los procedimientos odontológicos realizados a pacientes con discapacidad mental o psicomotriz, bajo anestesia general. Todos los casos fueron tratados en el quirófano mayor de la Clínica Dental Calafia, perteneciente a la Facultad de Odontología Mexicali, en la Universidad Autónoma de Baja California. Se investigó a partir de los informes quirúrgicos y los reportes clínicos, registrados en los expedientes clínicos del "Programa de Atención Odontológica a Pacientes con Discapacidad”, a pacientes con discapacidad mental y psicomotriz, que recibieron tratamiento dental bajo anestesia general, durante el periodo de tiempo comprendido desde enero de 2016 a diciembre de 2017.

De un universo conformado por 120 pacientes, atendidos bajo anestesia general en el quirófano de la FOM, se estudió una muestra intencional de 38 pacientes (31.7 \%) con discapacidad mental y/o psicomotriz que recibieron atención odontológica. Se utilizó el expediente clínico como fuente primaria para la obtención de los datos, que se registraron en un modelo de recolección diseñado al respecto. Se consideró como criterio de inclusión a todos los pacientes atendidos bajo anestesia general, con expedientes clínicos completos que tuvieron su consentimiento válidamente informado firmado; fueron excluidos, aquellos con datos incompletos o con otro tipo de discapacidad.

La operacionalización de las variables de estudio se organizó de la siguiente manera:

- Variables sociodemográficas: grupos de edades, medidas en años cumplidos, y sexo biológico.

- Variables clínico-terapéuticas: Tipo de discapacidad en dos categoría (grupo I mental y grupo II psicomotriz), padecimiento bucal con necesidad de tratamiento (caries, enfermedad periodontal y traumatismos), tratamientos realizados (restaurativo, en el que se incluían 
obturaciones con amalgama, resina o ionómero y coronas de acero cromo; quirúrgico que incluía extracciones y reducción de fracturas; pulpar para procedimientos de pulpotomía y/o pulpectomías; y por ultimo periodontal, a quienes se les realizó profilaxis y detartraje). La duración del procedimiento se conformaron con base en el periodo de tiempo (grupo A con duración de hasta dos horas y grupo B necesitaron procedimientos mayores de dos horas), para las complicaciones se evaluó si se presentaron (transoperatorias o postoperatorias). Por último, para clasificar las condiciones del paciente en la recuperación de la anestesia general se empleó la escala de recuperación de Aldrete modificada para cirugía ambulatoria medida del 1 al 10.

Para el análisis estadístico, se utilizaron estadígrafos descriptivos de frecuencia, porcentaje, media y moda; así como se aplicó la chi cuadrada $\left(\mathrm{X}^{2}\right)$ y el test exacto de Fisher para medir la asociación de variables $(\mathrm{p} \leq 0.05)$. La base de datos se estructuró, procesó y analizó con el paquete estadístico STATA versión 12. Se respetaron los principios bioéticos aplicables a este tipo de investigación documental en humanos, con respeto a la confidencialidad de los datos y al consentimiento informado de la FOM, que permite utilizar la información registrada en los expedientes con fines de investigación.

\section{RESULTADOS}

De los 38 pacientes atendidos en el "Programa de Atención Odontológica a Pacientes con Discapacidad", entre 2016 y 2017, el 68 \% tenía discapacidad psicomotriz y el $32 \%$ presentaba 
discapacidad mental. Todos necesitaron anestesia general para recibir tratamiento de sus padecimientos bucales.

En la Tabla 1, se muestra la distribución de los pacientes según grupos de edades y sexo biológico, en los que predominó el sexo masculino (68\%) sobre el sexo femenino (32\%). La media de edad fue de $15.71 \pm 8.75$ años, con límite inferior de 3 años y límite superior de 34 años, y el grupo de edades más representativo fue de 1 a 10 años con el $34.3 \%$ del total de pacientes.

TABLA 1

DESCRIPCIÓN DE LA POBLACIÓN ANALIZADA POR GRUPO DE EDAD Y SEXO

\begin{tabular}{cccc}
\hline $\begin{array}{c}\text { Grupo de Edades } \\
\text { Años }\end{array}$ & Fexenino & Masculino & $\begin{array}{c}\text { Total } \\
\text { n(\%) }\end{array}$ \\
\hline $0-10$ & 1 & 12 & $13(34.3)$ \\
$11-20$ & 6 & 5 & $11(28.9)$ \\
$21-30$ & 4 & 7 & $11(28.9)$ \\
$31-$ más & 1 & 2 & $3(7.9)$ \\
Total & 12 & 26 & $38(100)$ \\
$\overline{\boldsymbol{X}}=15.71 \pm 8.75$ & & & \\
\hline
\end{tabular}

Fuente* Expedientes clínicos de pacientes atendidos en la FOM

La Tabla 2, se aprecia la frecuencia de los padecimientos bucales con necesidad de tratamiento. Se identificó que todos los pacientes presentaron caries dental, como principal problema de salud bucal, y, en segundo orden, el $36.9 \%$ de los casos padeció de traumatismos dentoalveolares con compromiso dental. Ambas enfermedades orales, necesitaron atención odontológica especializada, bajo sedación anestésica, para prevenir las complicaciones derivadas de las mismas. 
TABLA 2

FRECUENCIA DE LOS PACEDIMIENTOS BUCALES CON NECESIDAD DE TRATAMIENTO

\begin{tabular}{ccc}
\hline Padecimiento Bucal & Frecuencia & $\%$ \\
\hline Caries Dental & 38 & 100 \\
Enfermedad Periodontal & 1 & 2.6 \\
Traumatismos & 14 & 36.9
\end{tabular}

Fuente* Expedientes clínicos de pacientes atendidos en la FOM

Se realizaron 53 procedimientos odontológicos que, según el tipo de discapacidad mental o psicomotriz, se reflejan en la Tabla 3. El $43.4 \%$ de los procedimientos fueron quirúrgicos (exodoncias y alveoloplastías) y el $41.5 \%$ consistió en tratamientos restaurativos conservadores (obturaciones y coronas de acero). Dada las condiciones de estos pacientes, fue necesario el uso de anestesia general, con un tiempo anestésico proporcional a la cantidad de enfermedades bucales presentes.

TABLA 3

FRECUENCIA DE PROCEDIMIENTOS REALIZADOS DE ACUERDO AL GRUPO DE DISCAPACIDAD

\begin{tabular}{|c|c|c|c|c|c|}
\hline \multirow{2}{*}{$\begin{array}{c}\text { Grupo de } \\
\text { Discapacidad }\end{array}$} & \multicolumn{5}{|c|}{ Procedimiento realizado } \\
\hline & Restaurativo & Quirúrgico & Pulpar & Periodontal & Totales \\
\hline $\begin{array}{c}\text { Mental } \\
\mathrm{n}=12(32 \%)\end{array}$ & 9 & 6 & 2 & 1 & 18 \\
\hline $\begin{array}{l}\text { Psicomotriz } \\
n=26(68 \%)\end{array}$ & 13 & 17 & 1 & 4 & 35 \\
\hline Total & $22(45.5 \%)$ & $23(43.4 \%)$ & $3(5.7 \%)$ & $5(9.4 \%)$ & $53(100 \%)$ \\
\hline
\end{tabular}


La Tabla 4 describe la distribución de los pacientes en función del tiempo de duración del procedimiento y la escala de recuperación de Alderete. Más de dos tercios de los pacientes necesitaron menos de 2 horas, bajo anestesia general, para recibir su tratamiento odontológico y el resto fue atendido con más de 2 horas de tiempo anestésico. Por otro lado, el $94 \%$ obtuvo de 9 a 10 puntos en la escala de recuperación de Alderete modificada.

TABLA 4

COMPLICACIONES Y ESCALA DE RECUPERACIÓN CON RESPECTO AL TIEMPO DE DURACIÓN DEL PROCEDIMIENTO

\begin{tabular}{lccc}
\hline & \multicolumn{2}{c}{ Escala Aldrete } \\
& \multicolumn{2}{c}{ Tiempo del procedimiento } \\
& & 9 a 10 & 8 \\
\hline Máximo 2 horas & $26(68 \%)$ & 26 & 0 \\
Más de 2 horas & $12(32 \%)$ & 10 & 2 \\
TOTAL & $38(100 \%)$ & $36(94 \%)$ & $2(6 \%)$ \\
\hline & Fuente* Expedientes clínicos de pacientes atendidos en la FOM
\end{tabular}

Desde una mirada analítica transversal, la Tabla 5 muestra que hubo asociación con significación estadística entre las complicaciones y el tiempo de duración del procedimiento bajo anestesia general mayor a 2 horas, con valor de $\mathrm{X}^{2}=7.05$ y un valor de $\mathrm{p}=0.029$ para una prueba de $\mathrm{p} \leq 0.05$. El resto de los análisis bivariados, realizados entre las complicaciones y otras variables, no fueron estadísticamente significativos. 
ASOCIACIÓN DE LAS COMPLICACIONES TRANS Y POSOPERATORIAS POR TIEMPO DE DURACIÓN DEL PROCEDIMIENTO.

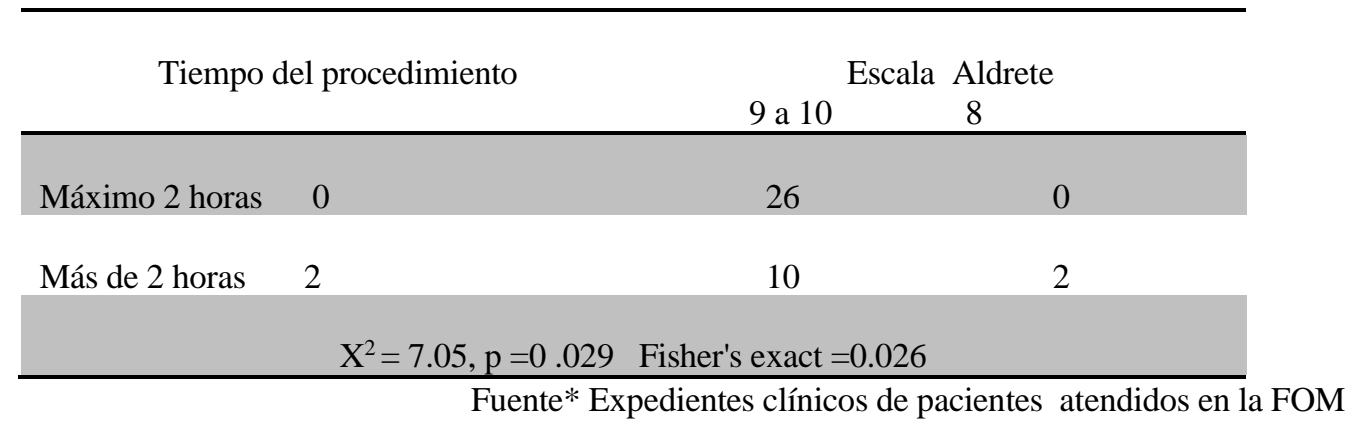

\section{DISCUSIÓN}

De acuerdo con los reportes del estado de Baja California, sobre la situación epidemiológica de las discapacidades, entre los pacientes atendidos por el "Programa de Atención Odontológica a Pacientes con Discapacidad”, entre 2016 y 2017, en la Facultad de Odontología Mexicali, de la Universidad Autónoma de Baja California; predominaron las discapacidades psicomotrices ante las mentales. En otros estudios, con tamaños de muestra muy similares, otros autores como Pérez "et al" (19), encontraron diferencias en cuanto al tipo de discapacidad atendida, pues predominaron las discapacidades mentales sobre las psicomotrices y esto se relaciona con el contexto geográfico (espacial y temporal) en que se realice la investigación.

Desde una perspectiva sociodemográfica, la mayor incidencia en el sexo masculino con respecto al sexo femenino, y la alta necesidad de atención odontológica en edades pediátricas inferiores a los 10 años, ha sido reportada por otros autores como Wang “et al” (17), Ebtissam (18), Pérez “et al” (19), 
Suárez "et al" (20) y Lara-Rojas "et al" (21). En los pacientes con discapacidad, las necesidades de atención odontológica durante la dentición mixta aumentan, por las irregularidades en el recambio dentario y la presencia de focos infecciosos en la dentición temporal, que pueden afectar la dentición permanente, por tal motivo, hay un incremento de pacientes entre los 6 a los 10 años. En otros contextos, tanto dentro como fuera de México, existen diferencias en la frecuencia de discapacidades según el sexo, pero lo que resultó valioso para esta investigación fue la caracterización de la población que acude a la FOM, de la UABC.

En cuanto a las necesidades de anestesia general para la atención odontológica de pacientes con discapacidad, esta es una medida terapéutica altamente recomendada por toda la literatura consultada. Las enfermedades bucales más frecuentes fueron las caries y las fracturas dentoalveolares por daño crónico, lo que coincide con Pérez "et al” (19), Lara-Rojas A "et al” (21), Oliveira del Rio "et al" $(22,23)$ y Mehmet "et al" (24). Otros investigadores, como Oliveira del Rio "et al” (23), encontraron que las extracciones dentales fueron más necesarias que las restauraciones y determinaron otras enfermedades como maloclusiones y enfermedad periodontal.

La asociación entre las complicaciones derivadas de la atención odontológica y el tiempo de duración del procedimiento, bajo anestesia general, mayor a 2 horas, ha sido reportada por otros investigadores como Wang "et al" (17) y Ebtissam (18). Ambos autores mencionaron la evidente relación entre la presencia de complicaciones, sobre todo postoperatorias, y el tiempo de atención odontológica, bajo anestesia general, superior a 2 horas; con énfasis en que ninguna de las complicaciones ocasionó problemas críticos relacionados con el estado circulatorio o respiratorio del paciente, algo muy similar a lo observado en los casos de esta investigación. 


\section{CONCLUSIONES}

En los pacientes con discapacidad mental y psicomotriz, atendidos en la Facultad de Odontología Mexicali, predominó la discapacidad psicomotriz, con mayor incidencia en menores de 10 años y más frecuente en personas del sexo masculino. Todos los pacientes presentaron caries dental, que se acompañaba de fractura dentoalveolar en más de un tercio de los casos, por lo que hubo alta necesidad de tratamientos quirúrgicos. La frecuencia de complicaciones derivadas de la anestesia general fueron pocas y estuvieron asociadas al tiempo de exposición a la anestesia general.

\section{RECOMENDACIONES}

Concientizar a los cirujanos dentistas sobre la importancia de la promoción y educación para la salud, la prevención y el diagnóstico precoz de las enfermedades bucales en los pacientes con discapacidad.

Extender el "Programa de Atención Odontológica a Pacientes con Discapacidad" en poblaciones vulnerables, de escasos recursos y con dificultades para acudir a los servicios odontológicos.

Desarrollar actividades docentes, científicas y asistenciales durante la atención odontológica a pacientes con discapacidad.

\section{AGRADECIMIENTOS}


Universidad Autónoma de Baja California, Facultad de Odontología Mexicali, Dr, Luis Ismael del Castillo Mercado y Dr. Víctor Hugo Castillo Gutiérrez Anestesiólogos del Programa de Atención a Pacientes con Necesidades Especiales .

\section{REFERENCIAS}

1. Organización Mundial de la Salud. Discapacidad y salud 2018. [Internet]. [Consultado 3 Agosto 2019]. Disponible en: https://www.who.int/es/news-room/fact-sheets/detail/ disability-andhealth

2. Organización Mundial de la Salud. Clasificación Internacional del Funcionamiento, de la Discapacidad y de la Salud: CIF, 2001. [Internet]. [Consultado 3 Jul 2019]. Disponible en: https://aspace.org/assets/uploads/publicaciones/e74e4-cif_2001.pdf

3. NORMA Oficial Mexicana NOM-015-SSA3-2012, Para la atención integral a personas con discapacidad. [Internet]. [Consultado 8 Jul 2019]. Disponible en: http://dof.gob.mx/nota_detalle.php?codigo=5268226\&fecha=14/09/2012

4. Instituto Nacional de Geografía e Informática. La discapacidad en México, datos al 2014.Versión 2017. [Internet]. [Consultado 5 Jul 2019]. Disponible en: http://conadistransparencia.org/transparencia_focalizada/La_discapacidad_en_Mexico_datos_al_2014_Versi on_2017.pdf

5. Organización Mundial de la Salud. Informe mundial sobre la discapacidad 2011. [Internet]. [Consultado 4 Jul 2019]. Disponible en: https://www.who.int/disabilities/ world_report/2011/es/ 
6. Instituto Nacional de Estadística y Geografía. La discapacidad en México, datos al 2014. [Internet]. [Consultado 3 Jul 2019]. Disponible en: http://coespo.qroo.gob.mx/ Descargas/doc/DISCAPACITADOS/ENADID\%202014.pdf

7. Corcuera-Flores JR, Delgado-Muñoz JM, Ruiz-Villandiego JC, Maura-Solivellas I, MachucaPortillo G. Dental treatment for handicapped patients; sedation vs general anesthesia and update of dental treatment in patients with different diseases. Med Oral Patol Oral Cir Bucal. 2014; 19 (2): 70-76.

8. World Health Survey. Geneva, World Health Organization, 2002-2004 [Internet]. [Consultado 6 Jul 2019]. Disponible en:http://www.who.int/healthinfo/survey/en/

9. Martínez Menchaca HR, Treviño Alanís MG, Rivera Silva G. Guía para el cuidado de la salud oral en pacientes con necesidad de cuidados especiales de salud en México. Revista ADM. 2011; 68(5): $222-28$.

10. Priyadarshni Bindal1, Chai Wen Lin1, Umesh Bindal, Sher Zaman Safi, , Zahidah Zainuddin, Andreas Lionel. Dental Treatment and Special Needs Patients (SNPs): Dentist's Point of View in Selected Cities of Malaysia. Biomedical Research. 2015; 26(1): 152-56.

11. Lozada-Leidenz MS, Guerra ME. Alternativas de atención odontológica en niños y adolescentes con discapacidad intelectual. Revista de Odontopediatría Latinoamericana. 2012; 2(2): 40-51.

12. Vitale F, Egidi R. Criterios de alta en cirugía ambulatoria. Rev Argent Anestesiol. 2007; 65(6): 427-31.

13. Glassman P. A review of guidelines for sedation, anesthesia, and alternative interventions for people with special needs; Spec Care Dentist. 2009; 29(1): 9-16.

14. Lim M, Borromeo GL. The use of general anesthesia to facilitate dental treatment in adult patients with special needs. J Dent Anesth Pain Med. 2017; 17(2): 91-103. 
15. American Dental Association. Guidelines for the Use of Sedation and General Anesthesia by Dentists. [Internet]. [Consultado 18 Jul 2019]. Disponible en: https://www.ada.org/ /media/ADA/Member\%20Center/FIles/anesthesia_guidelines.pdf?la=en

16. American Academy of Pediatric Dentistry. Management of Dental Patients with Special Health Care Needs. [Internet]. [Consultado 16 Jul 2019]. Disponible en: https://www.aapd.org/research/oral-health-policies--recommendations/management-of-dentalpatients-with-special-health-care-needs/

17. Wang N, Zao Y. Retrospective study of dental treatment under general anesthesia of 62 disabled childrens and adolescents; Journal of Pekin University. 2018; 50(2): 293-99.

18. Ebtissam Z. Murshid. Characteristics and Dental Experiences of Autistic Children in Saudi Arabia: Cross-sectional Study. J Autism Dev Disord. 2011; 41:1629-1634.

19. Pérez Bejarano N, Alarcón González V, Ferreira Gaona M, Díaz Reissner C, Duré P, Andriotti N, Moreno D, Galeano L, Mora A, Insfrán L, Jara R. Estado de Salud Oral en Discapacitados Residentes en la Fundación Pequeño Cottolengo Don Orione, Paraguay: Int. J. Odontostomat. 2016; 10(1): 69-74.

20. Suárez Zafra D, Villar Francois G, Suárez Vázquez B, Francois Ramírez MC, Estrada Sánchez G. Necesidad de tratamiento estomatológico en niños discapacitados de La Habana. Revista de Información Científica. 2018; 97(4): 798-809.

21. Lara-Rojas A, Chuquimarca-Paucar B. Prevalencia de caries dental y su relación con el pH salival en niños y adolescentes con discapacidad intelectual. Ciencias Médicas (ODONTOLOGÍA). 2017; 3(1): 474-87

22. Oliveira del Rio J, Sandoval Pedauga S, Andrade Vera F. Caracterización clínicoepidemiológica de pacientes especiales con maloclusión. Pol. Con. 8. 2017; 2(6): 1298-1310 
23. Oliveira del Rio J, Macías-Velásquez Z. Caracterización clinicoepidemiológica de pacientes especiales con gingivitis crónica. Dom. Cien. 2017; 3(4):250-63

24. Mehmet Ziya Yılmaza, Aysun Çağlar Toruna, Burcu Başb, Hatice Duranb, Hızır İlyas Köseb, Halit Furuncuoğlub. Anesthetic management of dental procedures in patients with special needs: A retrospective analysis of 519 patients in North of Turkey. J. Exp. Clin. Med. 2014; 31: 21316. 